\title{
Structured Early Consultation with the Occupational Physician Reduces Sickness Absence Among Office Workers at High Risk for Long-Term Sickness Absence: a Randomized Controlled Trial
}

\author{
IJmert Kant • Nicole W. H. Jansen • \\ Ludovic G. P. M. van Amelsvoort . \\ Rudy van Leusden · Ate Berkouwer
}

Published online: 15 January 2008

(C) The Author(s) 2008

\begin{abstract}
Objective To examine the efficacy of structured early consultation among employees at high risk for future long-term sickness absence, in the prevention and/or reduction of sickness absence. The focus of the experiment was the timing of the intervention, that is, treatment before sickness absence actually occurs. Methods In the current prospective randomized controlled trial (RCT), employees at high risk for long-term sickness absence were selected based on responses to a 34-item screening questionnaire including demographic, workplace, health and psychosocial factors associated with long-term sickness absence ( $>28$ days). A total of 299 subjects at risk for future longterm sickness absence were randomized in an experimental group $(n=147)$ or in a control group $(n=152)$. Subjects in the experimental group received a structured early consult with their occupational physician (OP), in some cases followed by targeted intervention. The control group received care as usual. Sickness absence was assessed objectively through record linkage with the company registers on sickness absence over a 1 year follow-up period. Results Modified intention-to-treat analysis revealed substantial and statistically significant differences $(p=0.007)$ in total sickness absence duration over 1 year follow-up between the experimental (mean 18.98; SD 29.50) and control group (mean 31.13; SD 55.47). Per-protocol analysis additionally showed that the proportion of long-term sickness absence spells ( $>28$ days) over 1 year follow-up
\end{abstract}

IJmertKant $(\varangle)$ · N. W. H. Jansen · L. G. P. M. van Amelsvoort Department of Epidemiology, Maastricht University, Care and Public Health Research Institute (CAPHRI), P.O. Box 616, 6200 MD Maastricht, The Netherlands e-mail: IJ.Kant@epid.unimaas.nl

R. van Leusden · A. Berkouwer

ABN AMRO Arbo Services, Amsterdam, The Netherlands was significantly $(p=0.048)$ lower in the experimental $(9.1 \%)$ versus control group (18.3\%). Conclusions Structured early consultation with the OP among employees at high risk for future long-term sickness absence is successful in reducing total sickness absence.

Keywords Epidemiology - Occupational health intervention · Prevention · Sick leave

\section{Introduction}

Long-term sickness absence and work disability constitute considerable public health problems, with important consequences for individuals, their families, for workplaces, and for society [1]. Within the Western countries musculoskeletal complaints and psychological health complaints are highly prevalent $[2,3]$, and account for the majority of certified sick leave [4-11]. Both musculoskeletal and mental health complaints are associated with prolonged sickness absence spells and permanent work disability. In the Netherlands, musculoskeletal and mental health complaints accounted for $65.5 \%$ of permanent work disability in 2004 [12]. Stansfeld et al. [7] for example demonstrated the importance of psychiatric disorders as a cause of sickness absence in the Whitehall II study, where psychiatric disorders were the third most common cause of certified absences of 8-21 days in duration and the second most common certified cause of absence of over 21 days for women working in the public service sector.

Longer absences are associated with a reduced probability of returning to work [13-16]. Nieuwenhuijsen et al. [17] demonstrated in a cohort of Dutch employees on sick leave with common mental disorders that the diagnoses of 
depression or anxiety disorder were significant predictors of a longer time until occupational rehabilitation. Possible explanations for the difficulty in returning to work are the illness severity in this stage, and the fact that the disorder may be intertwined with the work situation, since work characteristics may have been partially causing the mental health problems [18]. Some studies show that the effectiveness of treatment and rehabilitation of employees on sick leave due to mental health problems is often limited [19-21]. Therefore, it is hypothesized that a preventive strategy aimed at early intervention of employees before sickness absence or disability occurs, may be more effective. Several preventive strategies may be considered. One strategy could consist of primary prevention, implying that for all employees exposure to risk factors of sickness absence is limited as much as possible. Although this approach is the basis of many occupational health legislation and guidelines, this approach is not feasible in many cases. A second strategy would be to select employees at high risk for health complaints based on symptom levels, for example with respect to levels of fatigue, and provide treatment specifically aimed at influencing these symptoms and assuming that sickness absence will then be positively influenced as well. Although such strategy would probably be effective in reducing complaints, this does not necessarily imply a reduction of sick leave. A third and more promising strategy, applied in the present study, would be to specifically select those employees with a high risk of future long-term sickness absence, where an intervention should be aimed at the diffuse and complex range of risk factors in the etiology of sickness absence. For employees selected according to this strategy, the basis of intervention is the timing of the intervention that is, treatment before sickness absence actually occurs. A prerequisite for this preventive strategy however, is the ability to identify employees with a high risk for disability before sickness absenteeism actually occurs. For this purpose a screening questionnaire, the so-called Balansmeter, was developed [IJ. Kant et al., unpublished data] based on data of the Maastricht Cohort Study [22]. Internal validation in the Maastricht Cohort Study and external validation in a prospective cohort of bank employees in the Netherlands revealed good predictive properties for future long-term sickness absence, or disability [IJ. Kant et al., unpublished data]. As such, the Balansmeter may constitute a sound basis for screening and the application of a preventive approach, in which employees at high risk for future sickness absence can be detected and offered early consultation to prevent or reduce future sickness absence and subsequent disability. The aim of this study was to examine the efficacy of structured early consultation among employees at high risk for future long-term sickness absence, as identified by the Balansmeter, in the prevention or reduction of sickness absence over a 1 year follow-up period by means of a RCT.

\section{Methods}

Design

A randomized controlled trial (RCT) was conducted in the occupational health setting. Employees at high risk for long-term sickness absence, as identified by the Balansmeter, were randomized to receive structured early consultation with the occupational physician (OP) or to be followed up in a control group. Randomization sequences were generated by an independent research assistant using computerized random number generators. Block randomization (block size 2) was used to ensure equal numbers in each group and smooth enrollment in the trial over time. The primary outcome concerns sickness absence, which was measured objectively over a 12-month follow-up period between two through 14 months after completion of the Balansmeter. This study complied with the Declaration of Helsinki. Informed written consent was obtained from all participants in the trial. The complete study was introduced by the occupational health service from the participating company. The OPs from this occupational health service carried out the consultation, through which the whole study is covered by the medical guidelines of the occupational health service, thereby ensuring all subjects' privacy.

\section{Procedure}

This study was conducted in a large banking company in the Netherlands. The total population of this company was in 2003 characterized by a mean sickness absence of $5.0 \%$ (4.0\% in men and 5.6\% in women). In 2003, a random selection of 9,863 of 28,000 employees of this company received the screening questionnaire, the so-called Balansmeter [IJ. Kant et al., unpublished data], at their home address. In the invitation letter, employees were asked to provide written informed consent, which covered the usage of the questionnaire data, company data on sickness absence, and the possibility to be part of the trial. After 2 weeks a reminder was sent to all non-respondents. Recruitment started in January 2003 and was completed in October 2003. In total, 4,950 employees $(50.2 \%)$ responded to the questionnaire.

The Balansmeter, specifically developed for employees with an office work environment, was used to identify the employees at high risk for future long-term sickness absence. The Balansmeter was developed and validated in 
the Maastricht Cohort Study [22], and captures 19 individual predictors for men and 22 for women. Due to overlapping in predictors between men and women, the Balansmeter captures 34 multiple-choice questions originating from domains of the work environment, e.g., working conditions, psychological job demands; characteristics of the private situation, e.g., having to care for a chronically ill family member, the occurrence of lifeevents in the past year; (mental) health status, e.g., depressed mood; demographic factors, such as age and gender, and sickness absence history. Using an algorithm based on the weighted factors of the individual items of the model, a total score can be calculated, with higher scores indicating a higher risk for future long-term sickness absence [IJ. Kant et al., unpublished data]. A cut-off point has been defined, giving priority to a high specificity. For men, this resulted in a specificity of the Balansmeter of $94.4 \%$ and a sensitivity of $49.4 \%$ to detect future long-term sick leave ( $>28$ days) during the next year. This corresponds with a Relative Risk (RR) for long-term sickness absence of 13.39 (95\% CI 8.89-20.15). For women the specificity was $94.7 \%$ with a sensitivity of $30.3 \%$, resulting in a RR for long-term sickness absence of 5.79 (95\% CI 3.75-8.94). Subjects were not only selected because of their current health status, although it is observed that subjects with a chronic condition are more prone to be selected by the Balansmeter.

For this RCT, employees were eligible if they scored above the predefined cut-off point of the Balansmeter, were not absent from work, not pregnant and not receiving treatment by the $\mathrm{OP}$ at the time of completing the Balansmeter. A total of 299 respondents fulfilled these criteria. Due to a data processing error, initially 327 respondents were randomized, including 28 respondents who were incorrectly classified as scoring above the cut-off point of the Balansmeter. These persons were excluded from the trial and explain the different numbers of employees allocated to the experimental and control group.

For the intention-to-treat analysis we had to exclude subjects on baseline criteria that became only apparent after randomization, that is, after randomization we had to exclude those absent from work at the start of follow-up on sickness absence (2 months after receiving Balansmeter), since early consultation targets at those not on sick leave. Also excluded were those who left the ABN AMRO organization at one point during the follow-up period, as no objective sickness absence data could be obtained for these subjects. Pregnant women were excluded to avoid counting absence spells specifically related to pregnancy leave. Furthermore, one person was excluded because she indicated to already receive treatment before the start of the trial.

In this study, also a modified intention-to-treat analysis was applied in which we additionally excluded those already receiving treatment by the $\mathrm{OP}$, and those who had sought external treatment in the time lag between completion of the screening questionnaire and the consult with the OP, because for these persons early consultation was no longer applicable.

For the per-protocol analysis we additionally excluded those employees in the experimental group who did not accept the invitation for the consult with the OP. As such, the per-protocol analysis will reflect the group for which it was succeeded to advance intervention before sickness absence could occur.

A flow diagram on patient recruitment, allocation and outcome assessment is presented in Fig. 1.

\section{Intervention}

Employees at high risk for long-term sickness absence were randomized in an experimental group receiving early consultation or in a control group receiving care as usual. The focus of the experiment was the timing of the intervention, that is, before sickness absence might actually occur, rather than the type of intervention. Employees in the experimental group were invited for an extensive, one to one and a halve hour, consultation with the OP. The OP was chosen as the expert for consulting with employees at high risk for long-term sickness absence, because this occupational group is specifically equipped for recognizing work-related and non-work-related conditions and their interactions, as well as equipped for realizing targeted referrals to specialized care. The average number of days between receipt of the Balansmeter at their home address and the consult with the OP was 79.01 days (SD 24.53). Individual Balansmeter results were sent to the OPs in advance of the actual consult with the employee. Structured early consultation was conducted according to a protocol, capturing several steps. The first step of the consult consisted of clarification of the main symptoms and complaints. This was done by going through the individual Balansmeter results and through the conduct of a social and medical anamnesis as well as an anamnesis of the private situation and work situation, e.g., job history, job content, working conditions and terms of employment. Following problem and symptom clarification, the next step in the consultation was to explain the relation between these symptoms and risk of future long-term sickness absence. The final step of the consult consisted of explaining and discussing the expectations and benefits of early treatment for the employee. This consult may then result in a targeted intervention focusing at the specific complaints presented by the employee. Targeted intervention may consist of various conventional treatments, ranging from additional sociomedical counseling by the OP to psychotherapy, 
counseling by a social worker, or specialized and/or intensified care for a specific disease. In the present RCT, 84 employees had a consult with the OP, another 14 received additionally treatment besides the consult with the $\mathrm{OP}$, as retrieved from questionnaires completed by the OPs. Participants in the control group were offered no research intervention. If employees in the control group asked for help or in case of sickness absence, they received care as usual from the occupational health service.

In an earlier pilot study by two OPs of the occupational health service, specific information about the target population, and way of consulting this particular group, was obtained. In the RCT, a total of 12 OPs participated. They received a 1-day training in the conduct of structured early consultations before the study started. Besides explaining the aim of the study, the training focused on the different steps in the protocol for structured early consultation as described above. Based on experiences from the pilot study, the training further specifically aimed at the attitude needed for specifically consulting with employees who present themselves with relatively mild complaints and not yet on sick leave. Therefore, special attention was paid to symptom clarification and awareness raising for this target group. Finally also tools and means for referral to specialized care following the consult were provided during this training.

\section{Outcome Measurement}

All information regarding sickness absence was measured through record linkage on an individual level with the company registers on certified sickness absence. Long-term sickness absence was defined as a sickness absence spell lasting for more than 28 consecutive calendar days. This definition was chosen because, in the Netherlands, after

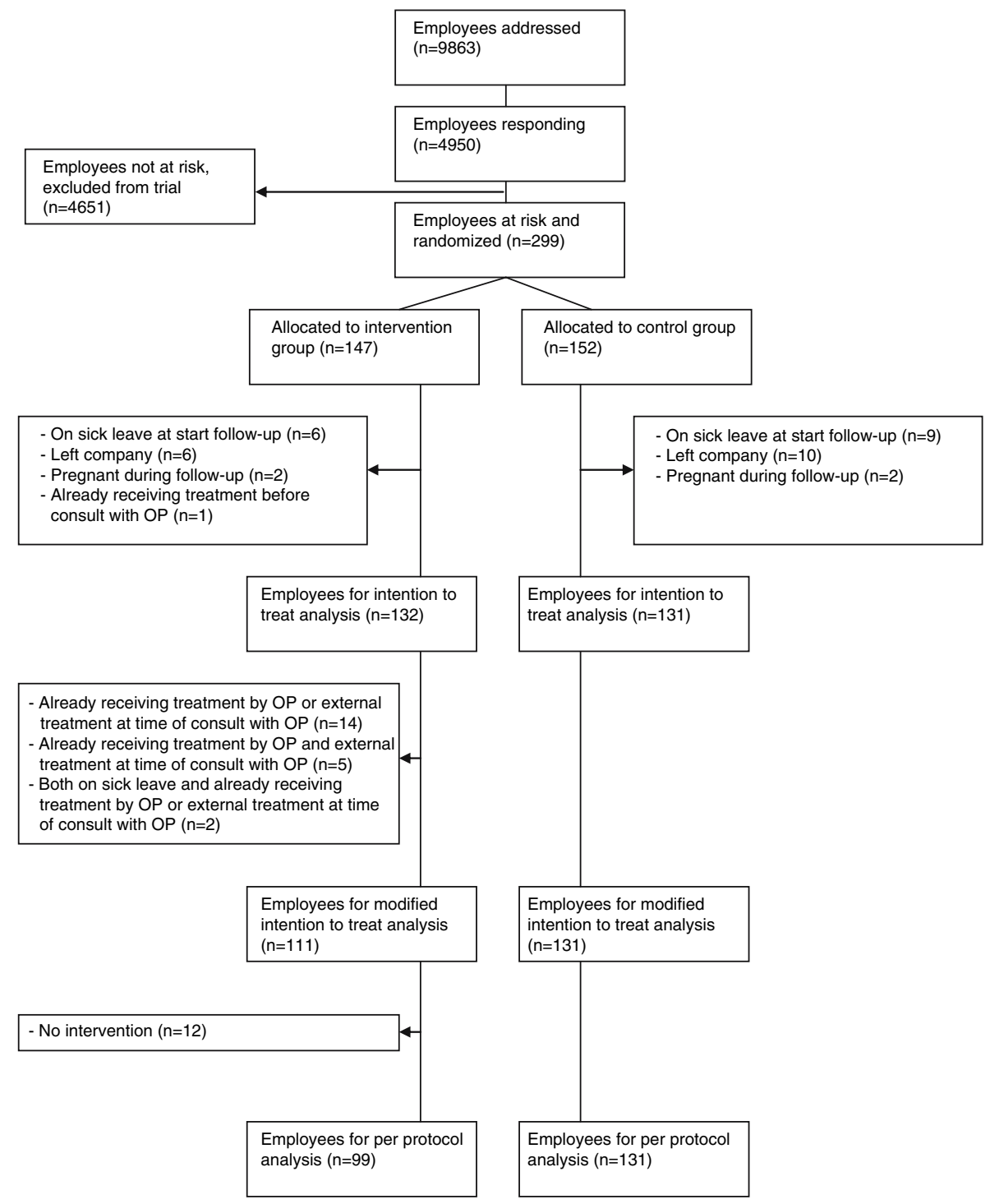

Fig. 1 Flow diagram of patient recruitment, allocation and outcome assessment 
28 days of sickness absence the employer is obliged to have completed a plan for reintegration and to report these employees to the sick leave insurance company. In line with the development of the Balansmeter, predicting sickness absence starting 2 months after completion of the questionnaire, the follow-up on sickness absence captured a 12-month follow-up period between two through 14 months after completion of the Balansmeter. Apart from the sickness absence data based on the company records, employees were asked in the Balansmeter to indicate whether they were absent at the time of completing the questionnaire. This information was used to exclude the employees absent from work at the time of completing the questionnaire.

\section{Statistical Analysis}

Based on the experiences within the Maastricht Cohort Study [22], we expected the incidence of long-term sickness absence of those scoring above the cutoff point of the Balansmeter to be $50 \%$ over a 1 year follow-up period. To reduce this rate from 50 to $35 \%$ (implying a $30 \%$ decrease), a power calculation before the study indicated that we would need 145 participants in both groups with a power of 0.9 and an alpha of 0.05. Data were analyzed on an intention-to-treat, modified intention-to-treat and per-protocol basis. Poisson regression analyses were used to test differences in sickness absence duration and sickness absence frequency. Other statistical procedures included Chi-square tests. All analyses were performed using SPSS and SAS.

\section{Results}

Baseline characteristics of all participants in the trial are displayed in Table 1. Age, mean number of years working for the company, and working hours per week were comparable in both groups. Small differences between the groups exist with respect to gender, educational level, and prevalence of conflicts with supervisor, depressed mood, the presence of a long-term illness, and the occurrence of life-events in the past year.

Table 2 shows differences in sickness absence over a 1 year follow-up period between the experimental and control group when analyzed according to the intention-totreat and modified intention-to-treat principle. When analyzed according to the intention-to-treat principle, participants in the experimental group showed a lower total sickness absence duration, lower sickness absence frequency, and lower proportion of long-term sickness absence spells as compared with the control group. Although relevant differences were observed, these differences were not statistically significant. The intentionto-treat analysis in the present study provides a more conservative estimate of the effectiveness of early consultation by including participants who were randomized for consultation but did not fulfill the criteria for early intervention. The modified intention to treat analysis, in contrast, revealed substantial and statistically significant differences in total sickness absence duration between the experimental and control group over 1 year follow-up.

Table 3 shows differences in sickness absence over a 1 year follow-up period between the experimental and control group when analyzed according to the per-protocol principle. As shown in Table 3, substantially and statistically significant differences were observed with respect to sickness absence duration and the proportion of long-term sickness absence spells between the experimental and control group, with an almost twofold higher number of total number of sickness absence days over 12 months follow-up in the control group versus the experimental group. These analyses were also stratified for gender. In
Table 1 Baseline characteristics of the study participants

\begin{tabular}{lll}
\hline & $\begin{array}{l}\text { Experimental } \\
\text { group }(n=132)\end{array}$ & $\begin{array}{l}\text { Control } \\
\text { group }(n=131)\end{array}$ \\
\hline Gender, \% male & 73.5 & 68.7 \\
Age, mean (SD) & $46.32(8.40)$ & $46.58(8.28)$ \\
Educational level & & \\
$\quad$ Low & 40.5 & 49.6 \\
$\quad$ Medium & 48.1 & 34.9 \\
$\quad$ High & 11.5 & 15.5 \\
Years working for company, mean (SD) & $23.87(10.96)$ & $23.54(11.13)$ \\
Working hours/week, mean (SD) & $34.37(3.95)$ & $33.90(4.77)$ \\
Conflicts with supervisor (\%) & 5.3 & 8.6 \\
Depressed mood (\%) & 17.6 & 20.6 \\
Long-term illness $(\%)$ & 54.5 & 47.5 \\
Life-events in the past year (\%) & 58.3 & 61.5 \\
\hline
\end{tabular}


Table 2 Differences in sickness absence (calendar days) over 12 months follow-up between experimental and control group according to intention-to-treat analysis and modified intention-to-treat analysis

\begin{tabular}{llll}
\hline Intention-to-treat analysis & Experimental group $(n=132)$ & Control group $(n=131)$ & $p$-value \\
\hline Total sickness absence duration (SD) & $25.97(44.84)$ & $31.13(55.47)$ & 0.290 \\
Sickness absence frequency (SD) & $2.40(2.04)$ & $2.60(3.06)$ & 0.500 \\
\% Long-term sickness absence ( $>28$ days) & 13.6 & Control group $(n=131)$ & 0.300 \\
\hline Modified intention-to-treat analysis & Experimental group $(n=111)$ & $31.13(55.47)$ & 0.007 \\
\hline Total sickness absence duration (SD) & $18.98(29.50)$ & $2.60(3.06)$ & 18.3 \\
Sickness absence frequency (SD) & $2.25(1.91)$ & 10.8 & 0.256 \\
\% Long-term sickness absence $(>28$ days) & 10.8 & & 0.102 \\
\hline
\end{tabular}

Table 3 Differences in sickness absence (calendar days) over 12 months follow-up between experimental and control group according to perprotocol analysis

\begin{tabular}{llll}
\hline & Experimental group $(n=99)$ & Control group $(n=131)$ & $p$-value \\
\hline Total sickness absence duration (SD) & $17.36(28.25)$ & $31.13(55.47)$ & 0.003 \\
Sickness absence frequency (SD) & $2.17(1.90)$ & $2.60(3.06)$ & 0.178 \\
\% Long-term sickness absence ( $>28$ days) & 9.1 & 18.3 & 0.048 \\
\hline
\end{tabular}

men, substantial differences were observed between the experimental versus control group with respect to total sickness absence duration [mean 18.12 (SD 30.91) versus mean 26.38 (SD 42.74); $p=0.090$ ], although these results just failed to reach statistical significance. In women, substantially and statistically significant differences were observed with respect to sickness absence duration with an almost threefold higher number of total number of sickness absence days over 12 months follow-up in the control group versus the experimental group [mean 41.56 (SD 75.98) versus mean 15.23 (SD 19.28); $p=0.021]$.

The per-protocol analyses for total sickness absence duration were also stratified for educational level. Nonsignificant differences in sickness absence duration were observed between the experimental and control group in the groups of employees with a low educational level [mean 14.98 (SD 23.54) versus mean 23.80 (SD 37.53); $p=0.106]$ or a high educational level [mean 9.14 (SD 13.02) versus mean 13.35 (SD 16.67); $p=0.506]$. Regarding employees with a medium educational level substantial and statistically significant differences were found between the experimental and control group [mean 20.72 (SD 33.08) versus mean $50.22(\mathrm{SD} 79.61) ; p=0.003]$.

\section{Discussion}

The aim of this study was to evaluate the efficacy of early consultation in an occupational health setting for employees at high risk for future prolonged sickness absence in a
RCT. To our knowledge, this is the first study examining the timing of intervention in relation to future long-term sickness absence. The RCT revealed substantial differences between the experimental and control group with respect to total sickness absence duration in favor of the experimental group. Stratified analyses for gender and educational level were also conducted, since they are often reported to be associated with sickness absence [23, 24]. In general, sickness absence duration was highest among women and among those with medium educational level. However, also the reduction in sickness absence was highest in the experimental group of these strata.

Screening employees at risk for future long-term sickness absence was done by means of the Balansmeter. The Balansmeter is capable of identifying the workers at risk, since in this study population the risk for long-term sickness absence was significantly higher for the selected employees as compared to the non-selected employees. For men the Relative Risk for long-term sickness absence was 3.90 (95\% CI 2.35-6.45) and for women 2.62 (95\% CI 1.44-4.77) [IJ. Kant et al., unpublished data]. It should be noted that this is especially remarkable given the fact that the sickness absence percentage in the total company was rather low at the time of study, which is in general negatively associated with the positive predictive value of a screening instrument. It should be noted however, that due to the response rate of $50 \%$ many employees at risk could not be identified.

Relevant differences in sickness absence duration were already observed in the intention-to-treat analysis, and 
were about as high as we expected from the power calculation. However, these differences failed to reach statistical significance. This can be attributed to the lower incidence of sickness absence in this study population as compared to the population which was used for the development of the Balansmeter. The power calculation of this study was based on the latter incidence. Although the incidence of sickness absence in the current study was much lower than expected, the modified intention-to-treat and per protocol analyses revealed that sickness absence was substantially and significantly lower in the experimental group as compared to the control group.

Considerable differences in the efficacy of structured early consultation in reducing long-term sickness absence were observed when comparing the results of the intentionto-treat versus modified intention-to-treat and per-protocol analyses. Quite a few subjects in the experimental group had already sought treatment before the consult with the OP. These subjects were excluded from the modified intention-to-treat and per-protocol analyses because adding early intervention to an already ongoing treatment would probably have no surplus value, and in that case the expression early consultation would be no longer applicable. This exclusion criterion may have resulted in an overestimation of the results from the modified intentionto-treat and per-protocol analyses, if these employees would be characterized by a higher level of complaints. On the other hand, when employees in the control group also have sought treatment, resulting in beneficial effects, then the results of the modified intention-to-treat and per-protocol analysis may be underestimated again. This point further stresses that early consultation is only effective among those who have not sought treatment themselves yet and emphasize the need of a short(er) time lag between screening and consultation with the OP. In this RCT the time lag between completion of the Balansmeter and the consult with the OP was on average 79 days, of which the dispatch of questionnaires, monitoring response, reminders, checking informed consent, and subsequent data processing and randomization took about 42 days. In order to reduce the time lag between completion and the actual consult, an electronic web based version of the screening questionnaire is under development. This application may reduce the time lag by more than $50 \%$. Further research should indicate how this time lag can be further reduced.

It should be noted that in this study the per-protocol definition is relatively mild, implying that employees at least had a consult with the OP. Further research should investigate the optimal intervention for this population, where existing treatments may be adjusted to the mild level of complaints and/or fine-tuning of treatments with regard to different target populations, for example focusing on musculoskeletal or mental health complaints, or educational level.

Especially in early consultation, at the beginning of a help-seeking process of the employee, we expect the role of the physician in both the guidance towards treatment and treatment itself to be very large. Therefore, differences in treatment effect between the different OPs cannot be ruled out. Given the numbers of employees and OPs in this RCT, subgroup analyses to explore these potential differences could not be conducted however. This subject is an important source for improvement and further study.

A cost-benefit evaluation was not part of this study. However, based on the intention to treat analysis we calculated that the decrease in sickness absence days in this study was 681 days. Using the average labor cost in the study population of $€ 189$ per worker per calendar day, the profits of reduction of sickness absence in this study amounted to $€ 127,368$. As this study is an RCT, this amount saved can be attributed to $50 \%$ of the screened population. It remains important, however, to monitor the profits of reduction of sickness absence versus the costs in terms of for instance monitoring, screening and treatment costs, of which the treatment costs will capture the majority of expenditure. Also with regard to cost-benefit evaluations, it might be beneficial to adjust consultations to the relatively mild stage of complaints among employees at risk for sickness absence, and/or to focus on more specific target groups.

Efficacy of structured early consultation was evaluated among employees with an office work environment. Therefore, this study is not fully representative for the general working population, limiting the external validity of our findings. Still, we would argue that the disadvantages concerning the ability to generalize our findings do not outweigh the advantages of this study population and intervention. That is, the RCT was developed for use in the occupational health care setting, characterized by a case load of stress-related (mental) disorders, in contrast with specialized care, where patients present with more clearly defined disorders. At this time, separate Balansmeter modules are being developed for use in other work environments, including industry and health care, enabling the evaluation of structured early consultations in other settings.

In conclusion, while future studies should explore several aspects for further improvement, regarding more specific target groups, adjusted treatments and a swift start of early treatment after screening, the results of this study clearly indicate that structured early consultation with the $\mathrm{OP}$, aiming at employees with a high risk for future longterm sickness absence, appears to be a promising strategy for preventing and/or reducing sickness absence. 
Acknowledgments This study was financially supported by the Care and Public Health Research Institute (CAPHRI), Maastricht, the Netherlands, and ABN AMRO Arbo Services, Amsterdam, the Netherlands. The authors acknowledge the contribution of Dr. G. M. H. Swaen, Maastricht University, in the initiation of this RCT, Dr. U. Bültmann, currently employed at the University Medical Center Groningen, for her contribution in the first phase of this study, including data collection, and Mr. J. J. M. Slangen, Maastricht University, for data management.

Open Access This article is distributed under the terms of the Creative Commons Attribution Noncommercial License which permits any noncommercial use, distribution, and reproduction in any medium, provided the original author(s) and source are credited.

\section{References}

1. Marmot M, Feeney A, Shipley M, North F, Syme SL. Sickness absence as a measure of health status and functioning: from the UK Whitehall II study. J Epidemiol Commun H 1995;49:124-30.

2. Paoli P, Merllié D. Third European survey on working conditions. Dublin: European foundation for the improvement of living and working conditions 2001.

3. Ihlebaek C, Eriksen HR, Ursin H. Prevalence of subjective health complaints (SHC) in Norway. Scand J Pub Health 2002;30:20-9.

4. Tellnes G, Svendsen KO, Bruusgaard D, Bjerkedal T. Incidence of sickness certification. Proposal for use as a health status indicator. Scand J Prim Health Care 1989;7:111-7.

5. Nystuen P, Hagen KB, Herrin J. Mental health problems as a cause of long-term sick leave in the Norwegian workforce. Scand J Pub Health 2001;29:175-85.

6. Houtman ILD, Smulders PGW, Klein Hesselink DJ. Trends in arbeid 2002 [Trends in work]. Hoofddorp: TNO Arbeid 2002.

7. Stansfeld S, Feeney A, Head J, Canner R, North F, Marmot M. Sickness absence for psychiatric illness: the Whitehall II study. Soc Sci Med 1995;40:189-97.

8. Hensing G, Spak F, Alexanderson K, Allebeck P. Sick leave among women and the role of psychiatric disorder. Scand J Soc Med 1997;25:185-92.

9. Hemingway H, Shipley MJ, Stansfeld S, Marmot M. Sickness absence from back pain, psychosocial work characteristics and employment grade among office workers. Scand J Work Environ Health 1997;23:121-9.

10. Alexanderson KA, Borg KE, Hensing GK. Sickness absence with low-back, shoulder, or neck diagnoses: an 11-year follow-up regarding gender differences in sickness absence and disability pension. Work 2005;25:115-24.
11. Hensing G, Andersson L, Brage S. Increase in sickness absence with psychiatric diagnosis in Norway: a general population-based epidemiologic study of age, gender and regional distribution. BMC Med 2006;4:19.

12. Central Bureau of Statistics. Statistisch Jaarboek 2005 [statistical yearbook 2005]. Voorburg/Heerlen: Centraal Bureau voor de Statistiek 2005.

13. Henderson M, Glozier N, Holland Elliott K. Long term sickness absence. BMJ 2005;330:802-3.

14. Frank JW, Kerr MS, Brooker AS, DeMaio SE, Maetzel A, Shannon HS, Sullivan TJ, Norman RW, Wells RP. Disability resulting from occupational low back pain. Part I: What do we know about primary prevention? Spine 1996;21:2908-17.

15. Williams DA, Feuerstein M, Durbin D, Pezzullo J. Health care and indemnity costs across the natural history of disability in occupational low back pain. Spine 1998;23:2329-36.

16. Watson PJ, Main CJ, Waddell G, Gales TF, Purcell-Jones G. Medically certified work loss, recurrence and costs of wage compensation for back pain: a follow-up study of the working population of Jersey. Br J Rheumatol 1998;37:82-6.

17. Nieuwenhuijsen K, Verbeek JH, de Boer AG, Blonk RW, van Dijk FJ. Predicting the duration of sickness absence for patients with common mental disorders in occupational health care. Scand J Work Environ Health 2006;32:67-74.

18. Janssen N, van den Heuvel WP, Beurskens AJ, Nijhuis FJ, Schroer CA, van Eijk JT. The Demand-Control-Support model as a predictor of return to work. Int J Rehabil Res 2003;26:1-9.

19. Van der Klink JJ, Blonk RW, Schene AH, Van Dijk FJ. Reducing long term sickness absence by an activating intervention in adjustment disorders: a cluster randomised controlled design. Occup Environ Med 2003;60:429-37.

20. Huibers MJ, Beurskens AJ, Van Schayck CP, Bazelmans E, Metsemakers JF, Knottnerus JA, Bleijenberg G. Efficacy of cognitive-behavioural therapy by general practitioners for unexplained fatigue among employees: Randomised controlled trial. Br J Psychiatry 2004;184:240-6.

21. Brouwers EP, Tiemens BG, Terluin B, Verhaak PF. Effectiveness of an intervention to reduce sickness absence in patients with emotional distress or minor mental disorders: a randomized controlled effectiveness trial. Gen Hosp Psychiatry 2006;28: 223-9.

22. Kant IJ, Bültmann U, Schröer CAP, Beurskens AJHM, Van Amelsvoort LPGM, Swaen GMH. An epidemiological approach to study fatigue in the working population: The Maastricht Cohort Study. Occup Environ Med 2003;60(Suppl 1):i32-i9.

23. Lund T, Labriola M, Villadsen E. Who is at risk for long-term sickness absence? A prospective cohort study of Danish employees. Work 2007;28:225-30.

24. Allebeck P, Mastekaasa A. Risk factors for sick leave-general studies. Scand J Public Health 2004;32(Suppl 63):49-108. 